\title{
Series with complete resolution of left atrial appendage thrombi with apixaban in elderly patients
}

Sang Hoon Lee ${ }^{1}$ and Seongwook $\mathrm{Han}^{2}$

${ }^{1}$ Division of Cardiology, Department of Internal Medicine, SM Christianity Hospital, Pohang; ${ }^{2}$ Division of Cardiology, Department of Internal Medicine, Keimyung University Dongsan Medical Center, Daegu, Korea
Received: May 1, 2015

Revised : May 26, 2015

Accepted: June 15, 2015

\section{Correspondence to}

Seongwook Han, M.D.

Division of Cardiology,

Department of Internal Medicine,

Keimyung University Dongsan

Medical Center, 56 Dalseong-ro,

Jung-gu, Daegu 41931, Korea

Tel: +82-53-250-7404

Fax: +82-53-250-7034

E-mail: swhan@dsmc.or.kr

\section{To the Editor,}

Non-valvular atrial fibrillation (AF) increases the risk of strokes by approximately 5 fold [1]. The atrial thrombi associated with AF are seen within the left atrial appendage (LAA) in most cases (>90\%) [2]. Anticoagulation with a vitamin-K antagonist (VKA) is recommended to prevent thromboembolic complications and to resolve thrombi [3]. Since 2010, several new (novel) oral anticoagulants (NOACs) have been developed that directly inhibit thrombin or coagulation factor-Xa. Those have emerged as alternative prophylactic agents to VKA [4]. However, data on the effect of NOACs regarding atrial thrombi related to non-valvular $\mathrm{AF}$ is limited. We report the resolution of LAA thrombi in two patients with persistent AF by using apixaban.

A 78-year-old woman who had a long history of hypertension, which had been well controlled, presented with AF of an unknown duration. She underwent a 12 lead electrocardiogram (ECG) for preoperative screening for spinal surgery. She was found to be in persistent AF, and an ECG had not been performed for several years. The transthoracic echocardiogram (TTE) showed a thickened mitral valve with mild to moderate regurgitation and a normal left ventricular ejection fraction. But the etiology of the mitral regurgitation could not be clarified at that time. The modified short axis view revealed mobile thrombi $(1.22 \times 1.6 \mathrm{~cm})$ in the LAA (Fig. 1A). Her $\mathrm{CHA}_{2} \mathrm{DS}_{2}$ VASc score was 4. She was admitted to the hospital for intravenous heparinization concomitant to starting VKA since she was not on anticoagulation. Fractionated heparin was administered intravenously for 7 days with warfarin and the activated partial thromboplastin time was kept two to three times longer than the reference value. The patient was discharged from the hospital with a final international normalized ratio (INR) of 1.9, which was expected to be higher than 2 in the next day. However, the patient returned to the clinic 5 days later with an INR of 1.51. The follow-up TTE showed smaller but remaining thrombi in the LAA. We discussed the risk of prolonged duration of the ineffective anticoagulation without coverage of heparin, and the time to achieve the therapeutic INR could be unpredictable. We also discussed that NOACs have rapid onset of action, which provide immediate anticoagulation effects minimizing the duration of ineffective anticoagulation predictably. After that, we agreed to switch warfarin to apixaban ( $5 \mathrm{mg}$ twice daily). The transesophageal echocardiogram (TEE) performed 6 weeks after apixaban treatment showed complete resolution of the thrombi in the LAA without any clinical systemic thromboembolic complications (Fig. 1B). 

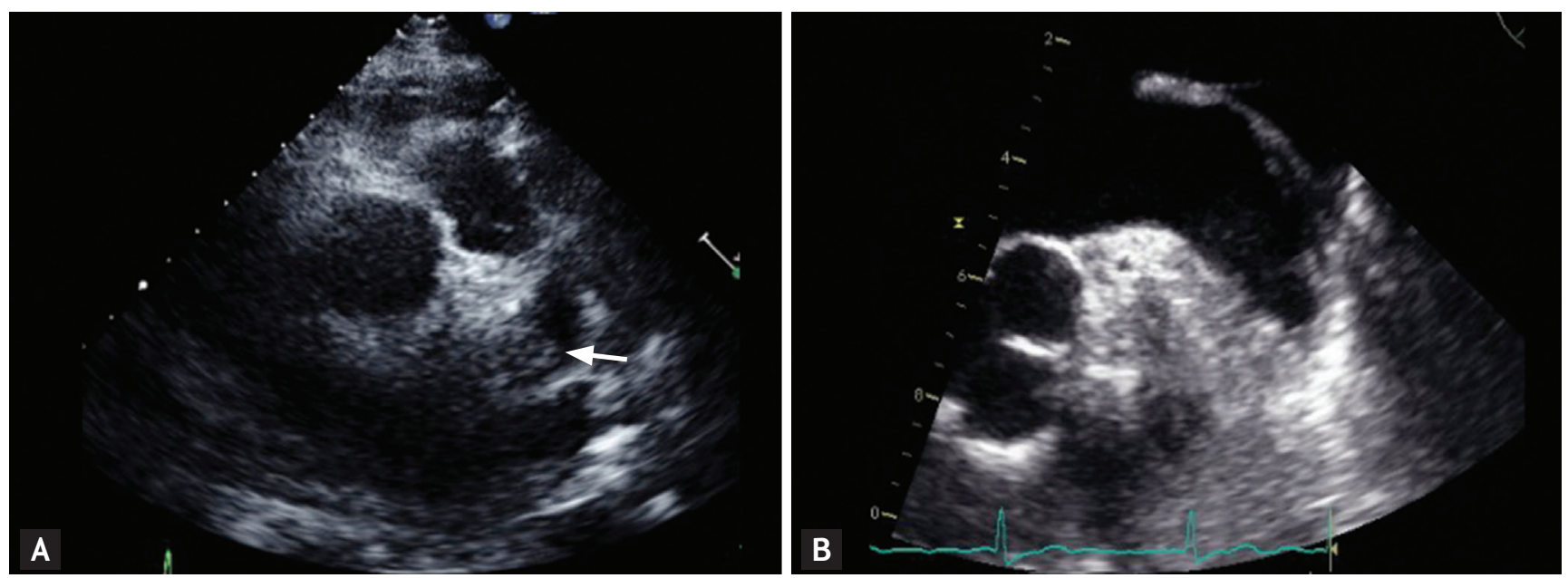

Figure 1. Echocardiographic studies in the first patient. (A) Modified short axis view of the transthoracic echocardiogram showing mobile thrombi (arrow) in the left atrial appendage. (B) Transesophageal echocardiogram 6 weeks after treatment with apixaban showing no evidence of thrombi in the left atrial appendage.
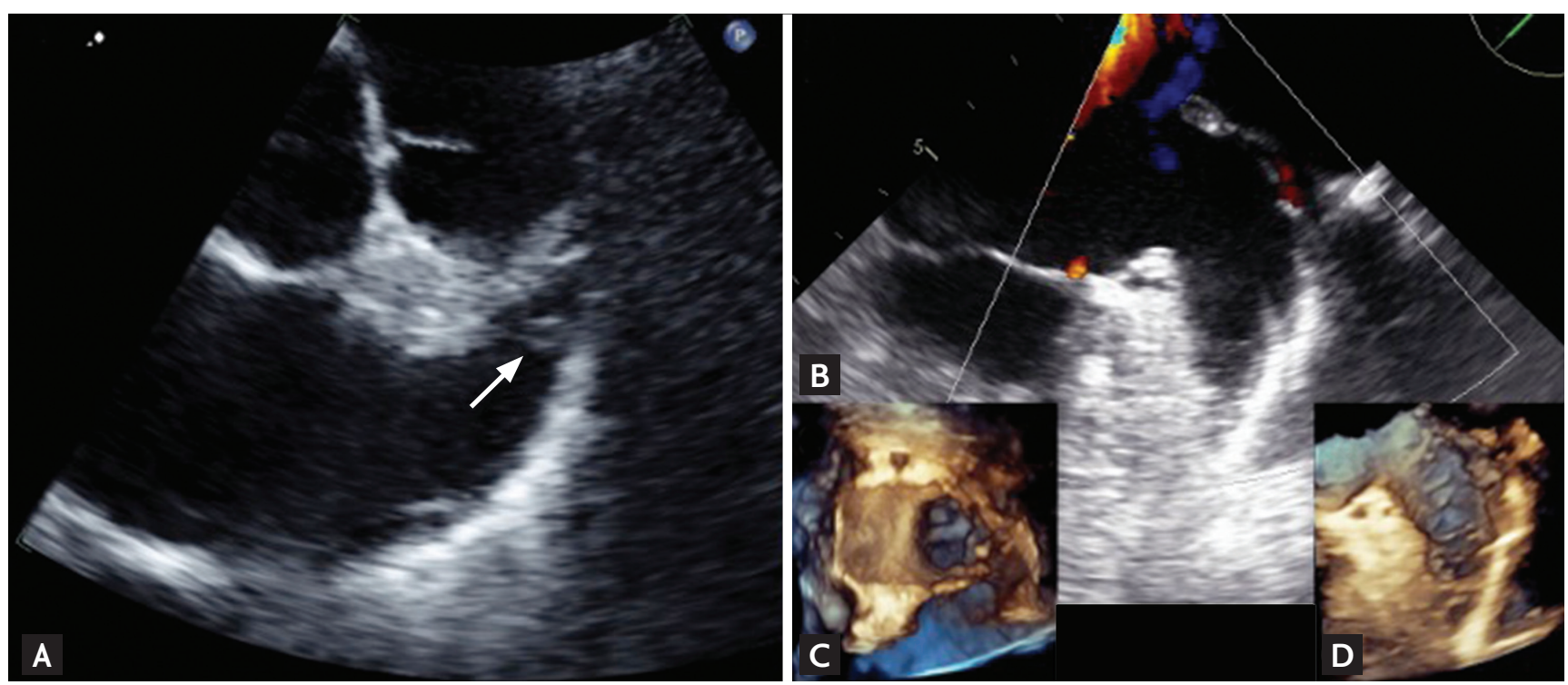

Figure 2. Echocardiographic studies in the second patient. (A) Modified short axis view of the transthoracic echocardiogram showing small thrombi (arrow) in the left atrial appendage. (B) Transesophageal echocardiogram showing no thrombi in the left atrial appendage, which was confirmed by 3-dimentional reconstructed images (C, en face view of the appendage; D, profile view of the appendage).

Another patient was a 79-year-old woman, who presented with aggravated dyspnea on exertion (DOE). She was also found to be in persistent AF, and the last available ECG (7 years ago) showed sinus rhythm. The $\mathrm{CHA}_{2} \mathrm{DS}_{2} \mathrm{VASc}$ score was 4 with hypertension. The short axis view of the TTE revealed small thrombi $(0.66 \times 0.51$ $\mathrm{cm}$ ) within the LAA (Fig. 2A). She was not on anticoagulant, either. The degree of her DOE was New York Heart Association class II to III, which did not need hospital- ization for treatment. We discussed that the predictable anticoagulation effects of NOAC, which could eliminate the concomitant heparin coverage that may need hospitalization. We shared the experience of the treatment result of the first patient. The patient and her family decided to choose NOAC. The apixaban (5 mg twice daily) was chosen as an anticoagulant and was prescribed from the outpatient clinic. The TEE after 5 weeks of treatment showed complete resolution of the thrombi in the LAA 
(Fig. 2B-2D).

VKA has a slower onset of action and the indirect anticoagulation activity depends on the clearance of the vitamin-K dependent clotting factors and dietary vitamin-K intake. The NOACs offer significant advantages over VKA with a rapid onset and offset of action, absence of an affect of diet on their action, and fewer drug interactions. Unresolved thrombi in the LAA could result in a subsequent higher risk of thromboembolic events [5]. In order to minimize the risk, it would be very important to resolve the thrombi as soon as possible.

Apixaban treatment effectively resolved the LAA thrombi and furthermore, shortened the time to achieve the effective anticoagulation than VKA, which could shorten the overall time to resolve the thrombi. Therefore, NOACs could be considered as the alternative anticoagulant of VKA for patients with non-valvular AF and atrial thrombi. Further investigation, in a larger population, of the efficacy of NOACs for resolving thrombi is warranted.

Keywords: Factor Xa inhibitor; Atrial appendage; Atrial fibrillation

\section{Conflict of interest}

No potential conflict of interest relevant to this article was reported.

\section{Acknowledgments}

We sincerely thank to Mr. John Martin for his linguistic assistance.

\section{REFERENCES}

1. Wolf PA, Abbott RD, Kannel WB. Atrial fibrillation as an independent risk factor for stroke: the Framingham Study. Stroke 1991;22:983-988.

2. Al-Saady NM, Obel OA, Camm AJ. Left atrial appendage: structure, function, and role in thromboembolism. Heart 1999;82:547-554.

3. January CT, Wann LS, Alpert JS, et al. 2014 AHA/ACC/ HRS guideline for the management of patients with atrial fibrillation: a report of the American College of Cardiology/American Heart Association Task Force on Practice Guidelines and the Heart Rhythm Society. J Am Coll Cardiol 2014;64:e1-e76.

4. Ruff CT, Giugliano RP, Braunwald E, et al. Comparison of the efficacy and safety of new oral anticoagulants with warfarin in patients with atrial fibrillation: a meta-analysis of randomised trials. Lancet 2014;383:955-962.

5. Corrado G, Tadeo G, Beretta S, et al. Atrial thrombi resolution after prolonged anticoagulation in patients with atrial fibrillation. Chest 1999;115:140-143. 